Revista de Psicología de la PUCP. Vol. XXI, 1, 2003 Edición de aniversario

\title{
Contenidos Rorschach en una muestra peruana
}

\author{
Matilde Ráez ${ }^{1}$ \\ Pontificia Universidad Católica del Perú
}

\begin{abstract}
Se investigó el área de Contenidos Rorschach en 237 habitantes de Lima. Los Contenidos constituyen un indicador para la comprensión de características básicas de la personalidad (auto-percepción, relaciones interpersonales, mediación cognitiva e ideación). El estudio fue descriptivo con muestreo probabilístico accidental. Las variables fueron edad, género, nivel de escolaridad. Se utilizó estadística descriptiva para analizar contenidos y variables demográficas, y no paramétrica (Kruskall-Wallis) para comparar datos. Los resultados destacan la ausencia de contenidos religiosos (Rl) y la importancia de contenidos anatómicos (An) en todas las variables. Los géneros difieren significativamente: los hombres interesados en la cultura y afán de logros y las mujeres, en el hogar. En cuanto a nivel de escolaridad, el grupo de educación superior y secundaria completa obtiene signos de solidaridad, interés en la socialización y mayor riqueza cognitiva.
\end{abstract}

Palabras claves: Psicodiagnóstico de Rorschach, contenidos, personalidad, género, nivel de escolaridad.

\section{Rorschach contents in a Peruvian sample}

This study stressed the Rorschach-Content area in 237 Lima inhabitants. The Contents are an indicator that helps to understand the basic characteristics of personality (self-perception, interpersonal relations, cognitive mediation and ideation). This descriptive study used an accidental-probabilistic sample. The variables were age, gender, schooling, The descriptive statistic was used to analyze demographic contents and variables, and the non-parametric statistic (Kruskall-Wallis) to compare data. The results stresses the absence of religious contents (RI) and the importance of anatomic contents (An) across the variables. The variable gender shows differences: men are interested in culture and show achievement motivation. Women are interested in home. With regarded to schooling, the group including superior educated and complete secondary educated students shows solidarity signs, interest in socialization and a high cognitive level.

Key words: Rorschach Psychodiagnostic-test, contents, personality, gender, schooling.

1 Doctora en psicología por la Katholieke Universiteit Nijmegen (Holanda). Es Profesora Principal del Departamento de Humanidades de la Pontificia Universidad Católica del Perú.. Sus trabajos de investigación comprenden estudios sobre género, pobreza y violencia y desarrollo evolutivo incluyendo el ciclo vital, así como evaluación y pruebas psicológicas, especialmente el test de Rorschach, temas sobre los que ha publicado artículos en revistas nacionales e internacionales. Asimismo, se desempeña en la práctica privada como psicoterapeuta. 

El Sistema Comprehensivo de Exner se apoya en un método con sólidas bases psicométricas, lo cual hemos comprobado en estudios normativos previos que hemos llevado a cabo en el Perú (Ráez,1998; Ráez, Maguiña, Iza, Jara y Velásquez, 1999; Ráez, 2002). Ello nos permite reforzar la importancia de continuar este tipo de trabajos que constituyen el soporte del sistema, aportando la confiabilidad de las variables como un elemento consistente en los distintos estudios .

En esta oportunidad nos propusimos investigar sobre un área distinta, muy importante para el estudio de la personalidad y directamente relacionada con la cultura: los Contenidos.

Los Contenidos ofrecen un acceso directo a los intereses y preocupaciones actuales de los participantes, pues constituyen un indicador acerca de las elecciones temáticas que la persona proporciona como respuesta a los estímulos de las láminas. También simbolizan motivaciones y actitudes que pueden o no tener una expresión directa en la conducta, pero que revelan aspectos muy particulares de los individuos.

Dentro del Sistema Comprehensivo, los Contenidos interactúan con otros indicadores en la generación de información útil para la comprensión de características básicas de la personalidad, como son la Auto percepción, las Relaciones Interpersonales, la Mediación Cognitiva y la Ideación.

Su análisis no difiere del de los otros factores Rorschach, ya que se apoya sobre la base de los datos normativos que ubican al participante dentro de un grupo con conductas específicas, directamente relacionadas con el simbolismo del contenido. 


\section{Metodología}

Se trata de un estudio de carácter descriptivo, que busca presentar las características de los contenidos Rorschach y de tres códigos especiales: Agresivo (AG), Cooperativo (COP) y Mórbido (MOR) en una muestra de 237 habitantes de Lima. Escogimos estos códigos dado que en un estudio anterior habían demostrado su valor relacionado con contenidos (Ráez,1998). El muestreo empleado es de tipo no probabilístico accidental.

\section{Participantes}

El género (Figura 1) se encuentra balanceado, ya que 108, el $46 \%$ de la muestra está conformada por varones, mientras que 129, el $54 \%$ restante lo constituyen mujeres.

En relación con la edad (Figura 2), se divide en cuatro grupos. Cabe señalar que la distribución de los participantes refleja la realidad peruana, conformada en su mayoría por una población joven (INEI, 1998).

En cuanto al grado de instrucción (Figura 3), se considera educación primaria $24 \%$, con menos de 7 años de estudios; secundaria $45 \%$, técnica $6.1 \%$ y universitaria el $19.3 \%$, datos similares a los descritos en el último censo (1998) y que señalan aumento de la población en la secundaria, con más de 7 años de estudios, lo que demostraría un mejor planteamiento del sistema educativo nacional.

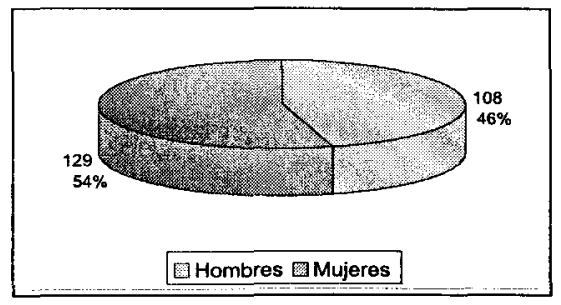

Figura 1. Distribución de la muestra por género. 


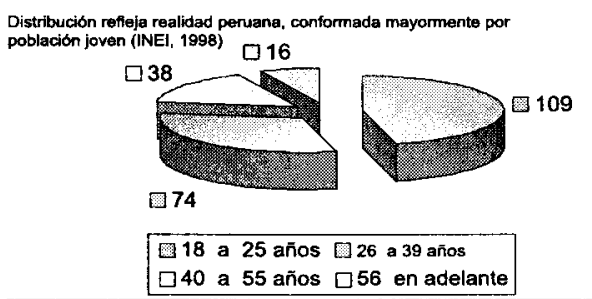

Figura 2. Distribución de la muestra por edad.

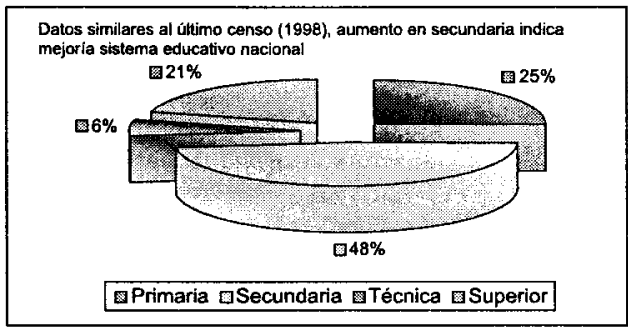

Figura 3. Distribución de la muestra por nivel educativo.

\section{Procedimiento}

El Rorschach es administrado y codificado de manera individual por el equipo que dirigimos y que llevó a cabo el estudio normativo de la prueba del que esta investigación forma parte (Ráez, Jara, Tejada y Plaza, 2003).

Se utilizó estadística descriptiva para analizar los contenidos de acuerdo a las variables demográficas de interés para este estudio. Para la comparación de datos se usó la estadística no paramétrica con el análisis de Kruskall-Wallis.

Para estudiar la calidad simbólica se dividieron los contenidos en tres grandes grupos siguiendo a Alcock, (1965) y a la escuela argentina: seres vivos, que implican las posibilidades de contacto y de 
interacción; amortiguadores, que señalan amplitud y variedad de contenidos, y también el tipo de enfoque para situar las diferentes problemáticas y perturbadores, que pueden orientar respecto a posibles áreas de conflicto (Cuadro 1).

\section{Cuadro 1}

Contenidos Simbólicos

\begin{tabular}{|ll|}
\hline & Contenidos \\
\hline Seres Vivos & $\mathrm{H}, \mathrm{Hd}, \mathrm{A}, \mathrm{Ad}, \mathrm{Hx}, \mathrm{COP}, \mathrm{An}, \mathrm{Sx}$, \\
Amortiguadores & $\mathrm{Art}, \mathrm{Ay}, \mathrm{Bt}, \mathrm{Cg}, \mathrm{Ge}, \mathrm{Hh}, \mathrm{Ls}, \mathrm{Na}, \mathrm{Sc}$ \\
Perturbadores & $\mathrm{Bt}, \mathrm{Cl}, \mathrm{Ex}, \mathrm{Fi}, \mathrm{Xy}, \mathrm{AG}, \mathrm{MOR}$ \\
\hline
\end{tabular}

\section{Resultados y Discusión}

Presentamos los hallazgos de la totalidad de Contenidos divididos según el tipo de respuesta.

Los contenidos humanos que representan aspectos del auto concepto, identidad y también de las relaciones interpersonales obtienen el nivel de representatividad esperada, aunque aumenta (Hd) y $\mathrm{Hx}$ en relación con estudios peruanos anteriores (Ráez et al., 1999 y Ráez, 2001 respectivamente). Indicarían cierta distancia y menor realismo en la conducta $((\mathrm{Hd}))$ y también esfuerzo por intelectualizar $(\mathrm{Hx})$ para neutralizar sentimientos dolorosos.

\section{Cuadro 2}

Resultados según Contenidos

\begin{tabular}{|lcccc|}
\hline Variable & Media & DE & Mínimo & Máximo \\
\hline $\mathbf{H}$ & 2.56 & 2.21 & .00 & 12.00 \\
$(\mathrm{H})$ & 1.39 & 1.41 & .00 & 7.00 \\
$\mathrm{Hd}$ & 1.38 & 1.49 & .00 & 9.00 \\
(Hd) & .87 & 1.19 & .00 & 6.00 \\
$\mathrm{Hx}$ & 0.57 & 1.03 & .00 & 6.00 \\
Suma H & 6.20 & 3.82 & .00 & 20.00 \\
\hline
\end{tabular}

$N=237$ 
Dada la importancia de los contenidos humanos ampliamos el estudio presentando una comparación de medias de los trabajos de Campo en España, y de Pires en Portugal ambos estudios de 1999, los países europeos más cercanos al nuestro en historia y en cultura. Destacamos que todos los signos de contenidos humanos tienen elementos estadísticos significativos, hecho que favorecería un estudio más en profundidad con un mayor número de datos para poder llevarlo a cabo.

De la comparación entre España y el Perú Hx en Perú se obtiene que los peruanos tendemos más hacia la intelectualización para intentar neutralizar nuestras emociones, en tanto que entre Perú y Portugal, los portugueses parecen presentar un mayor nivel de inhibición en sus contactos interpersonales. Los españoles resultarían con una mayor apertura hacia los vínculos y la socialización. Las posibilidades de un estudio multicultural se plantean (Cuadro 3).

\section{Cuadro 3}

Comparación de medias de Contenidos entre Perú, España y Portugal

\begin{tabular}{|c|c|c|c|c|c|c|c|c|}
\hline \multirow[t]{2}{*}{ Variable } & \multicolumn{2}{|l|}{$\begin{array}{l}\text { Perú } \\
N=23\end{array}$} & \multicolumn{2}{|c|}{$\begin{array}{l}\text { España } \\
N=520\end{array}$} & \multicolumn{2}{|c|}{$\begin{array}{l}\text { Portugal } \\
N=309\end{array}$} & \multirow{2}{*}{$\begin{array}{l}Z \\
\text { Peru- } \\
\text { España }\end{array}$} & \multirow{2}{*}{$\begin{array}{l}Z \\
\text { Perú } \\
\text { Portugal }\end{array}$} \\
\hline & Media & $D E$ & Media & $D E$ & Media & $D E$ & & \\
\hline $\mathrm{H}$ & 2.56 & 2.21 & 2.50 & 1.81 & 1.90 & 1.83 & 0.37 & $-3.72 * * *$ \\
\hline (H) & 1.39 & 1.41 & 1.40 & 1.28 & 0.79 & 0.97 & -0.09 & $5.61 * * *$ \\
\hline $\mathrm{Hd}$ & 1.38 & 1.49 & 2.16 & 1.97 & 1.25 & 1.70 & $-6.01 * *$ & 0.95 \\
\hline$(\mathrm{Hd})$ & .87 & 1.19 & 0.83 & 1.02 & 0.31 & 0.69 & 0.45 & $6.46 * * *$ \\
\hline $\mathrm{Hx}$ & 0.57 & 1.03 & 0.17 & 0.58 & 0.24 & 0.72 & $5.59 * * *$ & $4.21 * * *$ \\
\hline Suma H & 6.20 & 3.82 & 6.89 & 3.50 & 4.26 & 3.45 & $-2.36^{*}$ & $6.13^{* * *}$ \\
\hline
\end{tabular}

Nota. Significación a dos colas $* p<.05 \quad * * p<.01 \quad * * * \quad p<.001$

Ubicándonos nuevamente en el estudio peruano presentamos el resto de contenidos (Cuadros 4,5 y 6 ). El dato más significativo de estos cuadros, no lo constituye la presencia, sino la ausencia de un signo: el contenido Religioso. En estudios anteriores (Ráez, 1989, 
1991, 1998, 1999) su presencia era estadísticamente significativa. Incluso en nuestro trabajo de tesis doctoral (Raez, 1998) es un signo estructural de la personalidad según el análisis factorial, hallazgo que indica su importancia. En comunicación personal con el Dr. Exner (Boston, 1996), él señaló que dada su frecuencia debía ser incluido en las muestras normativas peruanas.

Éste es el primer estudio donde dicho contenido no tiene relevancia estadística ni cualitativa. Pareciera que el proceso de secularización que tiene lugar en América Latina también ha llegado al Perú, reduciendo la importancia de la religión como ayuda y seguridad y también del sentimiento mágico de escape asociado a ella, en el que desarrollo y esfuerzo son irrelevantes, puesto que el cambio viene de "arriba". Además, en la actualidad la crisis económica y de confianza en la autoridad se refleja en el desborde popular y el rechazo a simbolismos asociados con represión.

\section{Cuadro 4}

Resultados según Contenidos

\begin{tabular}{|lcccc|}
\hline Variable & Media & $D E$ & Mínimo & Máximo \\
\hline $\mathrm{A}$ & 7.79 & 3.35 & 2.00 & 21.00 \\
(A) & .42 & .76 & .00 & 4.00 \\
$\mathrm{Ad}$ & 2.36 & 2.00 & .00 & 12.00 \\
(Ad) & .12 & .36 & .00 & 2.00 \\
$\mathrm{An}$ & 1.34 & 1.61 & .00 & 9.00 \\
\hline
\end{tabular}

$N=237$

\section{Cuadro 5}

Resultados según Contenidos referidos a Códigos Especiales

\begin{tabular}{|lcccc|}
\hline Variable & Media & $D E$ & Mínimo & Máximo \\
\hline AG & .35 & .68 & .00 & 4.00 \\
COP & .60 & 1.03 & .00 & 6.00 \\
MOR & .97 & 1.30 & .00 & 6.00 \\
\hline
\end{tabular}

$N=237$ 
Contenidos Rorschach en una muestra peruana

Cuadro 6

Resultados según Contenidos

\begin{tabular}{|lcccc|}
\hline Variable & Media & $D E$ & Mínimo & Máximo \\
\hline $\mathrm{Art}$ & .79 & 1.12 & .00 & 6.00 \\
$\mathrm{Ay}$ & .49 & .79 & .00 & 4.00 \\
$\mathrm{Bl}$ & .24 & .61 & .00 & 4.00 \\
$\mathrm{Bt}$ & 1.30 & 1.31 & .00 & 7.00 \\
$\mathrm{Cg}$ & 1.71 & 1.56 & .00 & 8.00 \\
$\mathrm{Cl}$ & .16 & .45 & .00 & 3.00 \\
$\mathrm{Ex}$ & .14 & .36 & .00 & 2.00 \\
$\mathrm{Fi}$ & .49 & .76 & .00 & 4.00 \\
$\mathrm{Fd}$ & .27 & .64 & .00 & 6.00 \\
$\mathrm{Geo}$ & .09 & .30 & .00 & 2.00 \\
$\mathrm{Hh}$ & .93 & 1.02 & .00 & 6.00 \\
$\mathrm{Ls}$ & 1.01 & 1.28 & .00 & 6.00 \\
$\mathrm{Na}$ & .62 & 1.00 & .00 & 5.00 \\
$\mathrm{Sc}$ & 1.11 & 1.11 & .00 & 6.00 \\
$\mathrm{Sx}$ & .45 & .96 & .00 & 8.00 \\
$\mathrm{Xy}$ & .08 & .31 & .00 & 2.00 \\
$\mathrm{Id}$ & 1.09 & 1.56 & .00 & 11.00 \\
\hline
\end{tabular}

$N=237$

A continuación presentaremos los hallazgos más importantes según edad, género y nivel de instrucción y finalmente los elementos simbólicos que proporcionan los contenidos vivos, amortiguadores y perturbadores.

Edad.- (Figura 4) Sólo dos contenidos son significativos: Anatómico (An) en el grupo de 26 a 39 años y detalle parahumano (Hd) en el de 19 a 25 años.

El contenido anatómico en el rango de 26 a 39 años señala una mayor preocupación por el cuerpo asociada con cierto nivel de angustia referida a la sexualidad, en una etapa donde la pareja, la paternidad-maternidad y la búsqueda de logros laborales son los eventos más importantes en el proceso de desarrollo evolutivo. Por la relación con el esquema corporal quizá hubiéramos pensado que sería 
también frecuente entre los 19 y 25 años. Este hallazgo indicaría que el valor del cuerpo como símbolo de seguridad no es ya una necesidad básica sólo de la adolescencia, sino que continúa a través del ciclo vital asociando cuerpo, juventud y éxito como bases para cubrir las necesidades de logro.

(Hd) como contenido específico es sorprendente. Parecería que la fantasía y la distancia se acompañan de percepciones parciales de los otros dentro del proceso de interacción. Ello indicaría un mayor nivel de distancia en la socialización, con una visión poco realista de lo humano, dato que si bien corresponde a la ambivalencia adolescente, no se esperaría encontrar tan elevado en esta población. Creemos que para una interpretación más precisa se tendría que relacionar el dato con los otros signos del Rorschach.

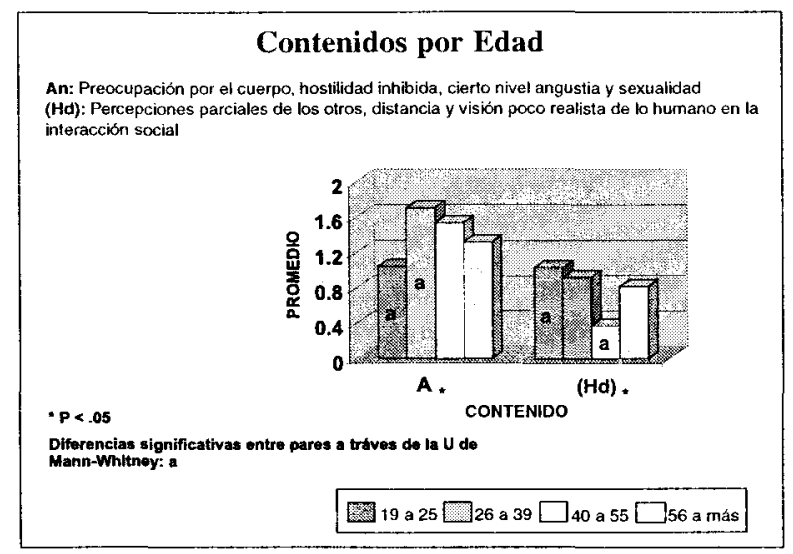

Figura 4. Contenidos significativos por edad.

Género.- El género en tanto construcción social del sexo, constituye un elemento central en la construcción de la identidad individual y social (Scott, 1990). El análisis de esta variable nos aproxima a las representaciones de lo masculino y lo femenino en nuestra sociedad (Figuras 5 y 6 ). 
Entre los hombres y las mujeres se han encontrado diferencias significativas en los siguientes contenidos: Ad, (Ad), Ay, Bl. Fd, Hh y MOR. Los hombres sobresalen en Ad, (Ad), Ay, Bl. Fd y MOR en tanto que las mujeres sólo en $\mathrm{Hh}$.

La impulsividad aparece en mayor medida en los hombres, to cual creemos se relaciona con el hecho de que su expresión es más permitida en los varones según las características asignadas a lo masculino en nuestra sociedad. También se encuentra presente un dato interesante, los intereses culturales indicadores de búsqueda de éxito profesional. También son los hombres quienes se presentan más pesimistas en el proceso ideacional anticipando de forma negativa su realidad, mientras que la combinación de Ad, (Ad) y Fd apuntaría hacia la dependencia afectiva e inhibiciones sociales relacionadas con inseguridad.

En las mujeres se encuentran los objetos domésticos aumentados (Hh). Estos contenidos referidos al hogar y a lo casero confirman resultados de estudios de género en el país en los que las mujeres se identifican con el espacio privado, restringiendo su orientación hacia el hogar (De Barnieri, 1996; Ráez,1998).

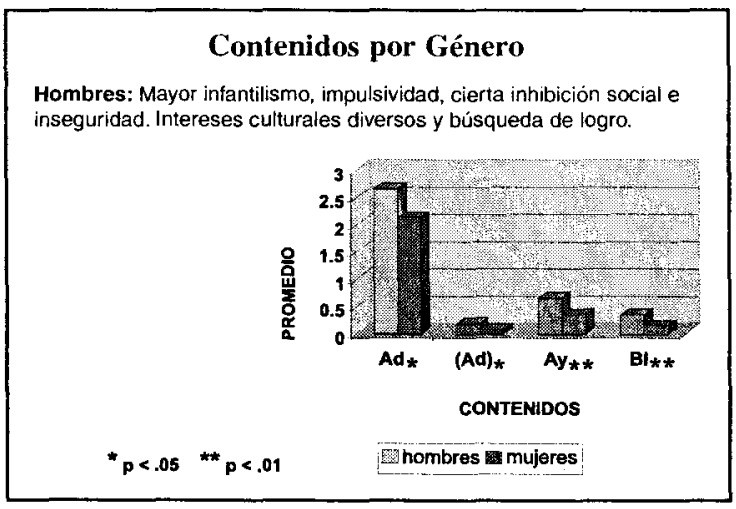

Figura 5. Contenidos significativos por género. 


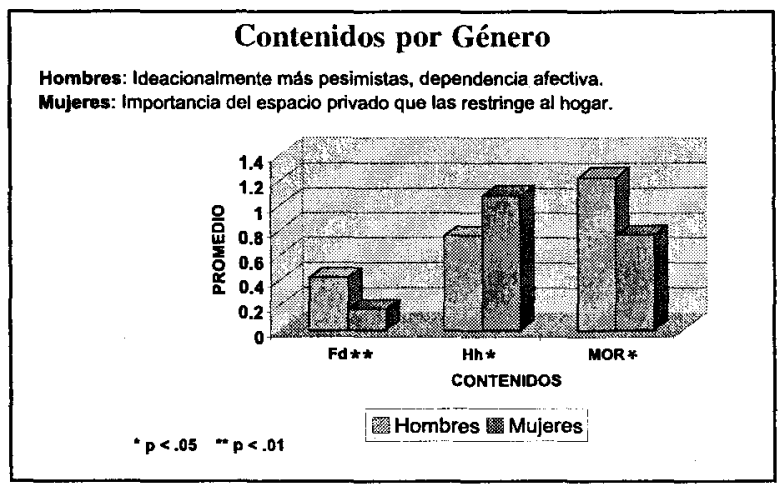

Figura 6. Contenidos significativos por género.

Nivel de Instrucción. Para una comprensión más amplia del tema, es importante relacionar esta variable con el nivel socio económico. Diversos estudios indican que en sectores poblacionales económicamente deprimidos el acceso a la educación superior es significativamente menor que en los estratos medio y alto (Martínez, 1999; Ráez, 1998). Además a mayor nivel educacional de los padres, son mayores los ingresos económicos y mejores los resultados escolares de los hijos.

Nuestros hallazgos siguen la línea de estos estudios. Se obtienen diferencias significativas (Figuras 7 y 8 ) en el ámbito interpersonal y en la auto percepción donde el interés por lo humano sobresale en las personas con instrucción superior y secundaria. En los participantes con instrucción escolar primaria, en cambio, el interés por los otros y la posibilidad de la empatía se encuentran disminuidos. Además, muy pocos en el grupo de menor instrucción perciben las relaciones humanas como cooperativas, lo que podría estar relacionado con una mayor desconfianza y suspicacia en dichas relaciones.

En los de nivel superior el valor de la educación significa un medio que estimula y refuerza las interacciones humanas, propiciando una 
auto percepción más positiva.(H). También sobresale una mayor sensibilidad a las formas sociales externas que se relacionan con estatus. Todas estas características implican una mejor elaboración conceptual y afán de logro. Sin embargo, es posible que al mismo tiempo fomenten la crítica en relación con los vínculos interpersonales y una cierta percepción distante y algo fantaseada del entorno $(H)$.

Un elemento de riesgo para las mujeres profesionales lo representaría el Hh alto, que encontramos en la variable género, porque sugiere que se podría producir un conflicto de intereses opuestos.

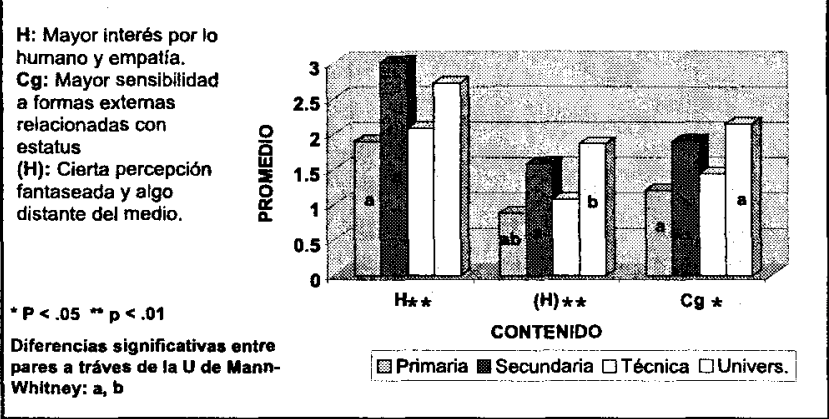

Figura 7. Contenidos significativos por nivel educativo.

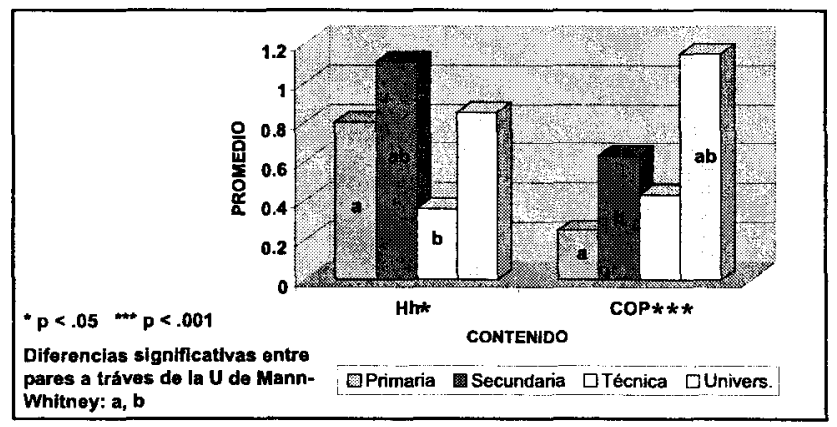

Figura 8. Contenidos significativos por nivel educativo. 
Contenidos Simbólicos. Presentamos las medias de los contenidos simbólicos, destacando el sesgo en relación de una presencia mayor en la cantidad de respuestas referidas a contenidos perturbadores (Figura 9).

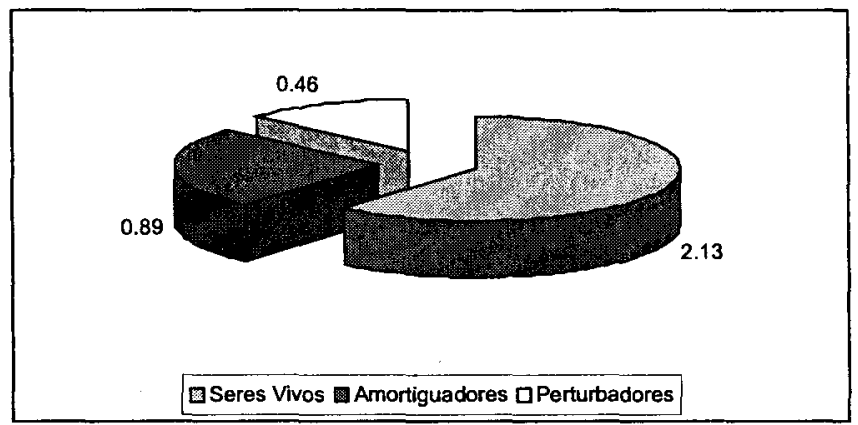

Figura 9. Promedio de grupos de contenidos simbólicos.

Dentro de la división de contenidos simbólicos (Figura 10) destaca en el grupo de contenidos vivos la presencia estadísticamente significativa de An. El contenido anatómico tiene para la muestra un valor relevante porque dentro del significado de seres vivos, indica que de las distintas posibilidades de contacto y de reconocimiento del entorno vital, eligen la única opción desvitalizada. Revisando el tipo de contenidos encontramos que prácticamente todos se referían a huesos, sólo dos respuestas indicaban interiores del cuerpo. Integrando estos datos, este hallazgo mostraría la exagerada preocupación por el cuerpo y la hostilidad relacionada con impotencia encubierta a través de la crítica y la ironía, que esconde frustración, cólera y susceptibilidad, rasgos en los que pensamos los peruanos podríamos reconocemos.

Ningún contenido amortiguador destaca en los hallazgos. Sin embargo, desde lo cualitativo sorprendentemente los 19 y 25 años y los 56 en adelante, obtienen resultados similares en Arte y en ropa 
(Cg). ¿La sensibilidad y el recubrimiento social en las etapas opuestas del desarrollo evolutivo?

Tampoco se obtienen datos estadísticamente significativos en los contenidos perturbadores. Cualitativamente cada etapa tiene un contenido particular destacando por su significado la impulsividad en los 26-39 a través de Ex y Fi y en los 40-55 las actitudes hostiles y el tono pesimista en la ideación de AG y MOR.

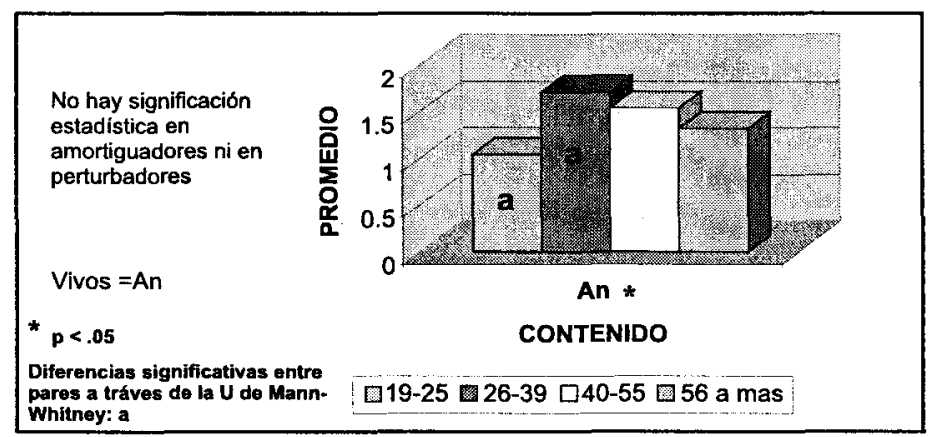

Figura 10. Contenidos simbólicos vivos por edad.

En cuanto al género (Figura 11) en seres vivos, sólo Ad. en los hombres resulta estadísticamente significativo. Dentro de la amplia gama de opciones más diferenciadas que representan los otros signos de este grupo, los participantes masculinos se identifican con Ad. Se trata del signo que simboliza justamente la actitud más infantil y fragmentada para la comunicación.

Desde lo cualitativo destaca la cooperación (COP) en las mujeres y el interés por la sexualidad en los hombres ( $\mathrm{Sx}$ )

Dentro de los amortiguadores sobresalen antropología (Ay) en los hombres y Objetos del Hogar (Hh) en las mujeres. Llama la atención que los hallazgos correspondan a los estereotipos. Desde el género los 
hombres se manejarían a partir de una mayor amplitud de intereses intelectuales y algo recubiertos y distantes afectivamente; mientras que las mujeres se sitúan ubicadas hacia lo práctico, con una orientación "de la casa", estando sus intereses bastante restringidos al espacio del hogar.

Los contenidos perturbadores sangre $\mathrm{Bl}$ y mórbidos $\mathrm{MOR}$ son significativos en los hombres. Ninguno lo es en las mujeres.

Los hombres muestran nuevamente una mayor libertad de expresión de sus contenidos simbólicos. Los hallazgos indican que el mayor riesgo de conflicto se encuentra en la dificultad para el manejo de aspectos agresivos que podrían manifestarse de manera directa o indirecta en hostilidad (Bl) y en el pesimismo en los procesos ideacionales (MOR).

Cualitativamente todos los signos son más elevados en los hombres. Es posible que a través de la inhibición y de la represión de los impulsos y en la pasividad, las mujeres escondan sus reales contenidos perturbadores.

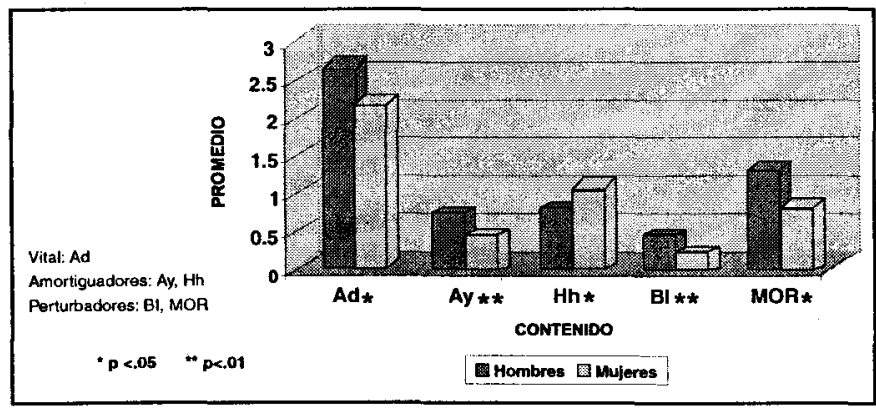

Figura 11. Contenidos simbólicos por género.

En cuanto a la educación (Figura 12), dentro de seres vivos, el contenido humano $\mathrm{H}$, la experiencia humana $\mathrm{Hx}$ y la conducta cooperadora 
COP son significativas en el nivel universitario. Tienen una percepción realista de sí mismos y de los demás, con opción a ser amables y cooperadores. En el lado opuesto los de educación primaria resultan los más distantes y menos dispuestos a la ayuda en las relaciones interpersonales.

En los contenidos amortiguadores, ropa $(\mathrm{Cg})$ y Doméstico $(\mathrm{Hh})$ son significativos. El primero $\mathrm{Cg}$ en el nivel universitario indicaría el interés por la apariencia, la sensibilidad y cierta fragilidad subyacente; en tanto que el grupo de secundaria es el más interesado en el espacio interior, del hogar.

Ningún contenido perturbador es estadísticamente significativo. Cualitativamente sobresale explosión (Ex), Fuego (Fi) y mórbidos (MOR) en el grupo Superior Técnico. Nos remiten a la dificultad para manejar la agresividad y a procesos ideacionales que se encuentran teñidos de pesimismo.

Una visión global del estudio señala la importancia en el Perú del nivel de instrucción, incluso sobrepasando en significados obtenidos al género y a la edad. El nivel educacional pareciera actualizar fenómenos psicológicos de mayor amplitud. Para la población estudiada el sistema valorativo, los deseos, las fantasías, temas centrales de la organización psíquica, difieren considerablemente si se trata de un grupo con instrucción primaria o universitaria. Por tanto, en el acceso a la educación es donde creemos que se grafica con mayor precisión la desigualdad y la marginación de los distintos grupos socio culturales.

En cuanto al género, llama la atención la diferencia de hallazgos en los hombres y en las mujeres. En los varones se encuentra impulsividad, infantilismo pero también intereses culturales y afán de logros. En cambio las mujeres se restringen a la importancia del hogar como espacio de interacción y la represión que esconde sus intereses y sus aspiraciones. ¿Se mantiene así el sistema machista? 


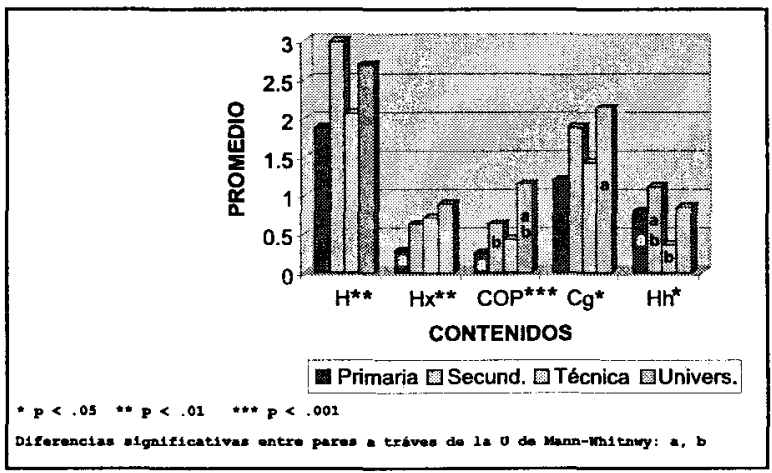

Figura 12. Contenidos simbólicos por nivel educativo.

\section{Reflexiones finales}

El análisis de los Contenidos es apasionante y a la vez difícil. Nos permite, como señala Chavert (1983) acercarnos a un área portadora de sentido y su articulación con las otras dimensiones de la respuesta Rorschach y abre el camino a una mayor comprensión a partir de la imagen que figura en la representación.

Desde lo multicultural representa de manera simbólica la variedad de opciones en los diferentes grupos, donde las imágenes personifican sus valores, deseos y aspiraciones particulares y resulta así un instrumento de evaluación muy valioso, en particular para un país tan fragmentado como el nuestro

A nivel de la investigación sin embargo, es quizá el área del Rorschach menos estudiada en la actualidad, tal vez porque se tiende a dar una importancia relativa al contenido como tal, y obviamente se priorizan los estudios sobre los determinantes, modos de enfoque, en general la variedad de mecanismos psíquicos que pone en juego las agrupaciones (clusters) de datos del sistema. 
Además, con los contenidos se corre el riesgo de error, de exagerar las relaciones simbólicas, con esa asociación vaga con lo psicoanalítico que sirve para apoyar muchas veces de manera pseudo científica cualquier interpretación, en la que todo es posible de asociar y de interpretar.

No obstante sus riesgos, pensamos que constituye un área que justamente por su ambigüedad y sutileza enriquece nuestro trabajo.

Estudios comparativos de contenidos en otras culturas nos acercarían a comprender sus intereses y sus sistemas de valores y también a las aspiraciones y a las fantasías con matices más cualitativos, que por el énfasis en las agrupaciones, estamos dejando de lado. Ello se vislumbra en el esbozo que hemos presentado en la comparación de algunos datos nuestros con otros similares de España y Portugal.

Esta investigación, por ejemplo, nos habla de cambios significativos en la población estudiada. La ausencia del contenido religioso abre un tema que es necesario evaluar desde una posición multidisciplinaria, pero que indica una modificación tanto en el plano ideacional, como en el sistema de valores.

En cambio, sorprende que se mantenga tan marcada la diferencia de enfoque desde el género y la presencia de sistemas simbólicos casi estereotipados, tales como el interés por la cultura en los hombres y la importancia del espacio reservado del hogar en las mujeres. Asímismo mientras que ellos pueden expresar impulsos y emociones hacia afuera, en ellas sobresalen por la inhibición, como si la realidad permitiera que lo masculino se exprese y lo femenino tenga que reprimirse.

La muestra total indica sensibilidad y preocupación respecto al cuerpo, eje de la identidad, así como hostilidad, irritabilidad, pesimismo, frustración que podrían representar la impotencia ante la realidad externa y la hostilidad se dirige hacia uno mismo. 
Finalmente, los hallazgos de la variable educación en la que los grupos de universitarios y de secundaria obtienen algunos de los signos más adecuados de la personalidad tales como solidaridad, interés genuino por la participación, mayor riqueza cognitiva, confirman la hipótesis con la que venimos trabajando desde hace más de una década (Ráez, 1985, 1989, 1998, 2001) que es a través de la educación que al estimular procesos del desarrollo evolutivo, se abrirían las posibilidades de modificaciones en aspectos básicos de la personalidad.

\section{Referencias}

Alcock, T. (1963). The Rorschach in practice. Londres: Tavistock. Alfaro, R. M., Rey, G. y Barbero, M. (1994). Entre públicos y ciudadanos: comunicación y cultura política. Lima: Calandria.

Balbi, C. R. (Ed.) (1997). Lima: Aspiraciones, reconocimiento y ciudadanía en los noventa. Lima: Fondo Editorial de la Pontificia Universidad Católica del Perú.

Baltes, P. B. (1987). Theoretical propositions of life-span developmental psychology: On the dynamics between growth and decline. Developmental Psychology, 23, 611-626.

Blondet Montero, C. (1993). Mujeres en el Perú. En T. Y. Valdés y E. Gomariz (Coords.), Mujeres latinoamericanas en cifras. Madrid: Instituto de la Mujer.

Campo, V. (1999) . An International symposium on Rorschach non patient data: Findings from around the world. Trabajo presentado en el XVI Congreso Internacional de Rorschach y Técnicas Proyectivas, Ámsterdam, Holanda.

Caspi, A. (1987). Personality in the life course. Journal of Personality and Social psychology, 53, 1203-1213.

Chavert, C. (1983). Le Rorschach en clinique adulte. Interprétation psychanalytique. París: Dunod.

De Barbieri, T. (1996). Los ámbitos de acción de las mujeres. En N. Henríquez.(Ed.), Encrucijadas del saber. Los estudios de género en las Ciencias Sociales. Lima: PUCP. 
Exner, J. E., Jr. (1993). The Rorschach: A comprehensive system: Basic Foundation (Vol. 1) (3ra. ed.). Nueva York: Wiley.

Fuller, N. (1993). Dilemas de la Femineidad. Mujeres de la clase media en el Perú (2da. ed.). Lima: Fondo editorial de la Pontificia Universidad Católica del Perú.

INEI (1998). Perú: compendios estadísticos. Lima: Dirección general de indicadores económicos y sociales.

Neugarten, B. L. (1968). The awareness of middle age. En B. L. Neugarten (Ed.), Middle age and aging (pp. 93-98). Chicago: University of Chicago Press.

Pires, A. (1999). An international symposium on Rorschach non-patient data: Findings from around the world. Trabajo presentado en el XVI Congreso Internacional de Rorschach y Técnicas Proyectivas. Ámsterdam, Holanda.

Raéz, M. y Martínez, P. (1985). Una aproximación a la violencia a través de los contenidos Rorschach en personal que labora en centros de readaptación. Revista de Psicología de la Pontificia Universidad Católica del Perú, 3 (2), 131-148.

Ráez, M., Martínez, P., Niño de Guzmán, I. y Rossel, Z. (1989). Avances para el estudio de la Identidad nacional peruana con el Rorschach para Grupos. Trabajo presentado en el VII Congreso Latinoamericano de Rorschach y otras técnicas proyectivas, Buenos Aires, Argentina.

Ráez, M. (1991). Le test collectiv de Rorschach comme instrument d'evaluation dans les études transculturelles. Rorschachiana, 17, 53-57.

Raéz, M. (1998). Personality Development of Women Leaders: Assessment studies in Peruvian Urban and Poverty areas. Tesis doctoral, Katholieke Universiteit Nijmegen, Holanda.

Ráez, M., Maguiña, D., Iza, M., Jara, L. y Velásquez, T. (1999). An international symposium on Rorschach non-patient data: Findings around the world. Trabajo presentado y tablas publicadas en el XVI Congreso Internacional de Rorschach y Técnicas Proyectivas, Ámsterdam, Holanda. 
Ráez, M. (2001). Hostilidad, agresión y violencia. Respuestas psicológicas y psicosociales. Trabajo presentado en el XXVIII Congreso Interamericano de Psicología, Santiago, Chile.

Ráez, M. (2001). El Rorschach para grupos. Un nuevo método para el estudio de la personalidad. Revista de la Asociación LatinoAmericana de Rorschach y Métodos Proyectivos, 6, 97-116.

Ráez, M., Jara, L., Tejada, P. y Plaza, B. (2003). Normas de Rorschach para población no-paciente. Hallazgos finales de investigación con poblaciones no pacientes alrededor del mundo. En prensa.

Scott, J. (1990). Género: una categoría útil para el análisis histórico. En J. Amelang y M. Nash (Eds.), Historia y Género. Las mujeres en la Europa moderna y contemporánea. Valencia: Alfons El Magnanim.

Weiner, I. (1995). Variable selection in Rorschach research. En J. E., Exner, Jr. (Ed.), Issues and methods in Rorschach research (pp. 73-9). Nueva York: Wiley. 